\title{
The Effect of Garlic Derivatives (S-Allylmercaptocysteine, Diallyl Disulfide, and S-Allylcysteine) on Gentamicin Induced Ototoxicity: An Experimental Study
}

\author{
Lokman Uzun ${ }^{1} \cdot$ Numan Kokten ${ }^{1} \cdot$ Osman Halit Cam ${ }^{2} \cdot$ M. Tayyar Kalcioglu ${ }^{1} \cdot$ M. Birol Ugur ${ }^{3} \cdot$ Muhammet Tekin $^{1}$ \\ Gul Ozbilen Acar ${ }^{1}$ \\ ${ }^{I}$ Department of Otorhinolaryngology, Head and Neck Surgery, Goztepe Training and Research Hospital, Istanbul Medeniyet University School of \\ Medicine, Istanbul; ' Department of Otorhinolaryngology, Head and Neck Surgery, Delta Hospital, Istanbul; \\ ${ }^{3}$ Department of Otorhinolaryngology, Head and Neck Surgery, Gazi University School of Medicine, Istanbul, Turkey
}

Objectives. Gentamicin is a potent aminoglycoside antibiotic. Ototoxicity and nephrotoxicity are the main side effects which restrict the use of gentamicin. Garlic with its intrinsic antioxidant activity may prove beneficial in prevention from ototoxicity. S-allylmercaptocysteine (SAMC), diallyl disulfide (DD), and S-allylcysteine (SAC) are three active compounds found in garlic. In this study, we investigated the effect of SAMC, DD, and SAC on the ototoxicity induced by gentamicin in rats, by using brainstem evoked response audiometry (BERA).

Methods. Thirty male Wistar rats with intact Preyer's reflex initially weighing 220-260 g were randomly assigned to either the gentamicin injection with SAMC treatment group (Genta-w SAMC), DD treatment group (Genta-w DD), SAC treatment group (Genta-w SAC), gentamicin injection without any active compounds (AC) treatment groups (Gentaw/o AC), or control group ( $\mathrm{n}=6$ rats each group). Gentamicin was given $120-\mathrm{mg} / \mathrm{kg}$ body weight, intraperitoneally once daily for 25 days to subjects in all groups except the control group. SAMC 100-mg/kg, and DD 50-mg/kg body weight were given intragastrically, and SAC $250-\mathrm{mg} / \mathrm{kg}$ body weight was given intraperitoneally once daily to subjects in Genta-w SAMC, and Genta-w DD, and Genta-w SAC groups, respectively during the study. After 25 days hearing thresholds were evaluated by using BERA test.

Results. The mean amplitude of auditory thresholds (sensation level [SL]) measured by using BERA for the Genta-w SAMC, Genta-w DD, Genta-w SAC, Genta-w/o AC, and control groups were $22 \pm 8,25 \pm 5,30 \pm 9,54 \pm 11$, and $10 \pm$ $7 \mathrm{~dB}$ SL, respectively (mean $\pm \mathrm{SD}$ ). The differences between every active compound group (Genta-w SAMC, Gentaw DD, and Genta-w SAC) and Genta-w/o AC were statistically significant $(P<0.016)$.

Conclusion. SAMC, DD, and SAC are derivative of garlic seems to attenuate aminoglycoside-induced hearing loss. The effect of SAMC and DD seems to be more prominent than that of SAC.

Keywords. Gentamicin; S-allylmercaptocysteine; Diallyl Disulfide; S-allylcysteine; Drug Toxicity

\footnotetext{
- Received June 27, 2015

Revised October 16, 2015

Accepted December 2, 2015

- Corresponding author: Lokman Uzun

Department of Otorhinolaryngology, Goztepe Training and Research

Hospital, Istanbul Medeniyet University School of Medicine, Dr. Erkin Cad.

Göztepe, Kadıköy, Istanbul,Turkey

Tel: +90-216-570-9075, Fax: +90-216-566-6614

E-mail: luzun99@yahoo.com
}

\section{INTRODUCTION}

Gentamicin is a broad-spectrum aminoglycoside antibiotic, which is commonly used worldwide. Though the development of many less toxic antibiotics, the aminoglycoside antibiotics are still indispensable in the treatment of some life threatening enterococcal, mycobacterial, and gram negative infections [1-5]. Cochlear, vestibular, and renal impairments are well-known

Copyright @ 2016 by Korean Society of Otorhinolaryngology-Head and Neck Surgery.

This is an open-access article distributed under the terms of the Creative Commons Attribution Non-Commercial License (http://creativecommons.org/licenses/by-nc/4.0)

which permits unrestricted non-commercial use, distribution, and reproduction in any medium, provided the original work is properly cited. 
complications [1]. These complications restrict the use of aminoglycosides. A potential therapeutic modality which would reduce or eliminate these complications, could enable more widespread use of the aminoglycosides [6].

The probable reason of aminoglycoside ototoxicity is inner ear damage by reactive oxygen species [4,7]. Reduction of ototoxicity obtained by cotherapy with free radical scavengers, antioxidants, or iron chelators supports this most widely accepted hypothesis [8]. Garlic (Allium sativum), garlic extracts, and some garlic constituents have intrinsic antioxidant activity. This antioxidant activity has been reported in in vivo and in vitro studies [9-11]. The active compounds of garlic (S-allylcysteine [SAC], Sallylmercaptocysteine [SAMC], diallyl disulfide [DD], alliin, and allicin) have antioxidant effects $[5,6,12,13]$.

Garlic and its purified constituents have been shown to protect against nephrotoxicity. The attenuator effect of gentamicininduced oxidative stress and nephrotoxicity of garlic [6], aged garlic extract [5], garlic oil [14], DD [12], SAC [13], and SAMC [15] have been demonstrated. It has been shown that, the reduction in gentamicin nephrotoxicity caused by garlic is not due to diminution of renal gentamicin concentration [6]. The effect of garlic on gentamicin induced ototoxicity has not been studied yet except our previous study [16]. We found that, garlic supplemented diet attenuates gentamicin induced ototoxicity in an experimental study. Garlic has many ingredients. The active compound, which is mainly responsible for attenuator effect of garlic gentamicin-induced ototoxicity, is not known and needs to be clarified. In this study, we have investigated the effect of SAMC, $\mathrm{DD}$, and SAC, which are active compounds of garlic on gentamicin-induced ototoxicity in rats by using brainstem evoked response audiometry (BERA).

\section{MATERIALS AND METHODS}

Thirty male healthy active Wistar rats, initially weighing 220 $260 \mathrm{~g}$ were used. Animals were randomly assigned to either the gentamicin injection with SAMC treatment group (Genta-w SAMC), DD treatment group (Genta-w DD), SAC treatment

\section{H I I G H L I I G H T T S}

- Ototoxicity is one of the main side effect which restrict the use of Gentamicin.

- Garlic with its intrinsic antioxidant activity may beneficial in prevention from ototoxicity.

- Garlic derivatives (S-allylmercaptocysteine [SAMC], diallyl disulfide [DD], and S-allylcysteine [SAC]) seems to attenuate gentamicin-induced hearing loss.

- The effect of SAMC and DD seems to be more prominent than that of SAC. group (Genta-w SAC), gentamicin injection without any active compounds (AC) treatment group (Genta-w/o AC), or control group ( $\mathrm{n}=6$ rats each group).

The investigation was approved by Ethics Committee of Marmara University Animal Center (DEHAMER) (protocol code: 72.2012.mar). This study was conducted in DEHAMER.

\section{Diet}

All of groups were fed with commercial rodent diet (standard laboratory rodent diet). All animals had free access to water and food. The amount of chow consumption was noted for each group. The health condition and diet of the rats was under strict control throughout the study.

\section{Animals}

The rats were supplied from DEHAMER. Animals were not accepted into the study unless they had bilateral normal Preyers' reflexes and translucent tympanic membrane upon endoscopic examination. Gentamicin was given $120-\mathrm{mg} / \mathrm{kg}$ body weight, intraperitoneally once daily for twenty-five days to subjects in Genta-w SAMC, Genta-w DD, Genta-w SAC, and Genta-w/o AC groups. SAMC $100-\mathrm{mg} / \mathrm{kg}$ and DD $50-\mathrm{mg} / \mathrm{kg}$ body weight were given intragastrically, and SAC $250-\mathrm{mg} / \mathrm{kg}$ body weight was given intraperitoneally, once daily to subjects in Genta-w SAMC, Genta-w DD, and Genta-w SAC groups, respectively during the study. In Genta-w/o AC group, rats were fed with standard rodent diet and were not treated with any of active compounds of garlic. Gentamicin and any of active compounds were not given to control group. Control group fed with standard rodent diet. All animals had free access to water and food. The weight of each rat was controlled at the beginning and the end of the study.

\section{Reagents}

Active compound of garlic (SAMC, DD, and SAMC) were obtained from Wakunaga Pharmaceutical Co. Ltd., Hiroshima, Japan. Gentamicin was from İ.E. Ulagay lliaç Sanayii Türk A.Ș., Istanbul,Turkey.

\section{Auditory threshold measurement}

Thresholds were determined for each animal on 25th day by using BERA. Rats were anaesthetized with ketamine $(100 \mathrm{mg} / \mathrm{kg})$ and klorpromazin $(5 \mathrm{mg} / \mathrm{kg})$ mixture given intraperitoneally as an induction anesthesia. Ketamin 35-mg/kg and klorpromazin $2-\mathrm{mg} / \mathrm{kg}$ mixture were given intramuscular as a maintenance therapy. Auditory thresholds were measured by using evoked auditory brainstem response. BERA recordings were performed in a sound-proof booth. In brief, click stimulus (20-msec duration, 1-msec fall time, low pass filter $100 \mathrm{~Hz}$, high pass filter 3 $\mathrm{kHz}$, and stimulus repetitions 1,500) generated using a Medelec Synergy EMG and EP Systems software ver. 11.0 (VIASYS Healthcare, Madison, WI, USA) and presented to ears via a 


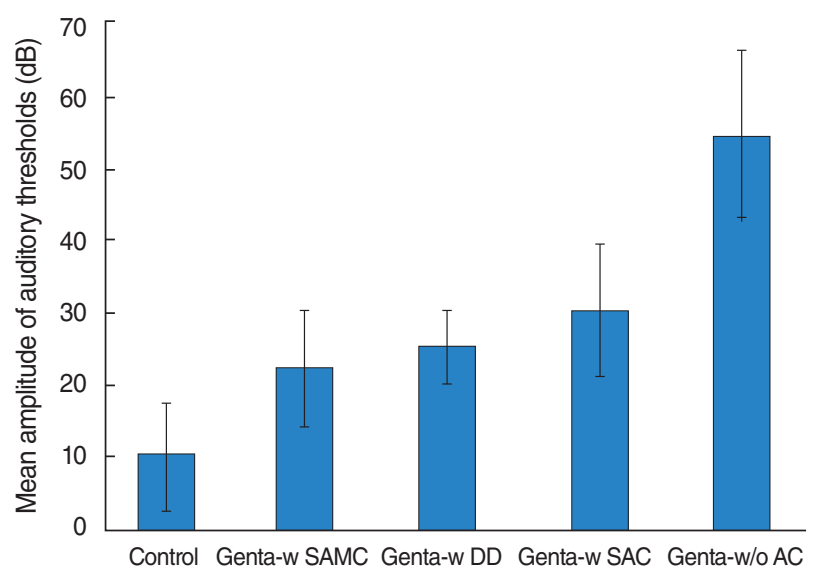

Fig. 1. The mean amplitude of auditory thresholds (sensation level) measured by using brainstem evoked response audiometry for the control, Genta-w SAMC, Genta-w DD, Genta-w SAC, and Genta-w/o AC groups. Genta-w SAMC, gentamicin injection with S-allylmercaptocysteine; Genta-w DD, gentamicin injection with diallyl disulfide; Genta-w SAC, gentamicin injection with S-allylcysteine; Gentaw/o AC, gentamicin injection without any active compounds (SAMC, $\mathrm{DD}$, and SAC) of garlic.

headphone-Telephonics TDH-49P (VIASYS Healthcare). The active electrode was placed at the vertex, in the midline of the scalp between the external auditory canals. The reference electrode was placed subcutaneously below the pinna of the left ear, and the ground electrode was inserted to the contralateral side. The average responses from 1,024 stimuli were obtained at 10$\mathrm{dB}$ intervals near threshold. The lowest stimulus level at which a positive waveform in the evoked response trace was evident was recorded as the threshold level. The BERA of each animal was performed and interpreted-without knowledge of the treatment history of rats-by 3 researchers (LU, NK, and MTK).

\section{Statistics}

Mean \pm SD were calculated for auditory thresholds of the three groups. Kruskal-Wallis test was used as a non-parametric test to compare all 5 groups for the statistical analysis. A $P$-value lower than 0.05 was required for statistical significance for KruskalWallis test. Bonferroni adjusted Mann-Whitney $U$-test was used for post hoc analysis in order to compare the groups as pairs. A $P$-value lower than $0.016(0.05 / 3)$ was required for statistical significance for Bonferroni adjusted Mann-Whitney U-test. IBM SPSS Statistics ver. 21.0 program (IBM Co., Armonk, NY, USA) was used for statistical analysis.

\section{RESULTS}

The amount of total chow consumed throughout the study was almost the same for each group. The weight of each rat was noted at the beginning and the end of the study. We did not detect any meaningful difference regarding the weight of each rat at
Table 1. The mean amplitude of auditory thresholds (sensation level) measured by using brainstem evoked response audiometry for the Genta-w SAMC, Genta-w DD, Genta-w SAC, Genta-w/o AC, and control groups

\begin{tabular}{lccccc}
\hline Subject & Control & $\begin{array}{c}\text { Genta-w } \\
\text { SAMC }\end{array}$ & $\begin{array}{c}\text { Genta-w } \\
\text { DD }\end{array}$ & $\begin{array}{c}\text { Genta-w } \\
\text { SAC }\end{array}$ & $\begin{array}{c}\text { Genta-w/o } \\
\text { AC }\end{array}$ \\
\hline 1 & 10 & 30 & 20 & 20 & 50 \\
2 & 0 & 30 & 30 & 40 & 60 \\
3 & 10 & 20 & 20 & 40 & 40 \\
4 & 20 & 20 & 20 & 20 & 70 \\
5 & 10 & 10 & 30 & 30 & 50 \\
6 & - & 30 & 30 & 30 & - \\
Mean \pm SD & $10 \pm 7$ & $22 \pm 8$ & $25 \pm 5$ & $30 \pm 9$ & $54 \pm 11$ \\
\hline
\end{tabular}

Genta-w SAMC, gentamicin injection with S-allylmercaptocysteine; Gentaw DD, gentamicin injection with diallyl disulfide; Genta-w SAC, gentamicin injection with S-allylcysteine; Genta-w/o AC, gentamicin injection without any active compounds (SAMC, DD, and SAC) of garlic.

the beginning and at the end of the study. Two animals were lost from Genta-w/o and control groups during anesthesia. All of the remaining animals underwent final hearing evaluation by using BERA and Preyer's reflex.

After the tenth day, hearing was evaluated by the Preyer's reflex daily. By the 25th day the reflex was absent in all members of Genta-w/o AC group animals but present in all control group, Genta-w SAMC, Genta-w DD, and Genta-w SAC group animals.

The mean amplitude of auditory thresholds (sensation level [SL]) measured by using BERA for the Genta-w SAMC, Gentaw DD, Genta-w SAC, Genta-w/o AC, and control groups were $22 \pm 8,25 \pm 5,30 \pm 9,54 \pm 11$, and $10 \pm 7 \mathrm{~dB}$ SL, respectively (mean \pm SD) (Fig. 1). Hearing thresholds are presented in Table 1. Kruskal-Wallis test was used as a non-parametric test to compare all 5 groups for the statistical analysis. The differences were statistically significant $(P=0.001)$.

Bonferroni adjusted Mann-Whitney $U$-test was used for post hoc analysis to compare the groups as pairs. There were significant differences between the Genta-w SAMC and Genta-w/o AC, Genta-w DD and Genta-w/o AC and between the Genta-w SAC and the Genta-w/o AC groups $(P=0.008, P=0.004$, $P=0.009$, respectively). A $P$-value lower than $0.016(0.05 / 3)$ was required for statistical significance for Bonferroni adjusted Mann-Whitney U-test. There weren't any significant differences found when we compared the three agents as pairs i.e., SAMC vs. DD, SAMC vs. SAC, and DD vs. SAC $(P>0.05)$.

\section{DISCUSSION}

In this study we found that all the three active compounds-i.e., SAMC, DD, and SAC-attenuated gentamicin ototoxicity compared to controls. None of the 3 compounds was superior to another regarding this attenuation effect. 
The main limiting factors of clinical usage of gentamicin are renal, cochlear, and vestibular complications. If a potential therapeutic option to reduce these complications could be found, gentamicin would become a more widely used antibiotic $[1,6]$. Attenuator effects of supplemented garlic diet on gentamicin induced renal damage, and ototoxic effect were proved in previous studies [6,16,17].

Aminoglycoside antibiotics forms a complex with oxidative properties by chelating iron and thus promote the formation of free radicals. It has been shown that free radicals are responsible for production of lesions in the hair cells, especially in the outer hair cells [3]. Aminoglycoside induced destruction of the sensory and supporting cells of the organ of Corti ensues starting with the outer hair cells of the lower turns and progressing to the apex, systematically $[1,2,18]$. It has been concluded that the generation of reactive oxygen species (ROS) is linked to ototoxicity. The aminoglycosides lead to the formation of ROS as observed in vitro as well as in cell culture studies. ROS is known to be very harmful to biological tissues via lipid peroxidation, DNA strand breaks, carbohydrate and protein damage, and deranged membrane-bound enzymes and receptors [2].

Prevention of gentamicin induced ototoxicity is maintained by either of two ways. Firstly, aminoglycosides react with iron to generate ROS. Protection against ototoxicity thus may be achieved by reducing the availability of iron by using chelators, such as deferoxamine and dihydroxybenzoate and these are shown to be effective in preventing from aminoglycoside ototoxicity. Secondly, antioxidants may be used protect the cochlea. They are shown to be effective against aminoglycoside ototoxicity in experimental animal studies, including lipoic acid, D-methionine, salicylates, and dihydroxybenzoate $[2,18]$.

Several in vivo and in vitro studies have shown the intrinsic antioxidant activity of garlic (Allium sativum), garlic extracts, garlic oil and some garlic constituents [6,9-14,19-21]. Little data is available from scientific studies concerning the bioavailability of garlic-derived compounds. The bioavailability of $S A C$ is $98 \%$ in rats [22]. Unfortunately, no pharmacokinetic data are available for SAMC. Three in vivo studies showed that oral administration of SAMC $(200 \mathrm{mg} / \mathrm{kg})$ decreased acetaminophen-induced liver injury in mice, and attenuates gentamicin-induced renal damage and thus indicating the bioavailability of orally administrated SAMC $[15,23,24]$. Diallyl sulfures and vinyldithiins are the major components of garlic oil and oil-macerate preparations. Vinyldithiins have been detected in the serum, kidney and fat tissue $>24$ hours after oral ingestion [19]. Synthesized S-labeled alliin, $60 \%-70 \%$ was absorbed in rats [21]. It was found that alliin along with DD could be detected in the perfusate after the isolated rat liver passage, but no allicin was found [25]. These findings indicate that alliin itself is never converted to allicin in the body and metabolized to various organosulfur compounds such as DD by liver enzymes [19].

Renal damage induced by gentamicin has been shown to be decreased by the garlic diet. The beneficial effect was not due to a decrease in renal gentamicin concentration, but rather was due to the prevention of the decrease of manganese-containing superoxide dismutase (Mn-SOD) and glutathione peroxidase (GPx) activities and the rise of lipoperoxidation in renal cortex observed in gentamicin nephrotoxicity [6]. Maldonado et al. [5] have shown in rats treated with gentamicin that aged garlic extract prevented the increase in carbonyl and nitrotyrosine levels in renal cortex. The effect could be associated with the antioxidant properties of aged garlic extract, and with its ability to prevent the decrease in antioxidant enzymes activity (Mn-SOD, GPx, and glutathione reductase). Therefore aged garlic extract contain compounds, which potentially ameliorate gentamicininduced nephrotoxicity. The compounds responsible of its protective effect are postulated to be S-allylcysteine, allymercaptocysteine, and alliin, which are water soluble, odorless, and have antioxidant properties [5].

The effect of active compounds of garlic on hearing has not been studied yet. In our previous study, we have shown that garlic supplemented diet attenuates the gentamicin induced hearing loss in animals [16]. In this study, we evaluated the protective effect of SAMC, DD, and SAC, which are the active compounds of garlic against gentamicin-induced ototoxicity. In the gentamicin injection with AC groups (Genta-w SAMC, Genta-w $\mathrm{DD}$, and Genta-w SAC), there was a significant reduction of ototoxicity detected by BERA compared to gentamicin injection without AC (Genta-w/o AC) group. Thus, treatment of garlic derivatives (SAMC, DD, and SAC) significantly attenuated aminoglycoside-induced hearing loss in rats as measured by Preyer's reflex and BERA. The effect of SAMC and DD seems to be more prominent than that of SAC but statistical comparison did not show a significant difference between their effects.

We detected that garlic derivatives (SAMC, DD, and SAC) have an attenuator effect in gentamicin-induced ototoxicity, possibly due to the antioxidant effect of garlic derivatives. The results of this study are rather exciting but it has several limitations. One of them is the small sample size. Secondly the beneficial effect of garlic needs to be shown by controlled human studies. Nevertheless such a simple measure like adding garlic to the diet may be of promise to prevent the irreversible ototoxicity due to aminoglycosides. Many patients may refuse to take garlic powder or fresh garlic due to their pungent odor [5]. Thus the route of administration may involve medical forms that would avoid the pungent odor.

BERA recordings in our study were performed using click stimulus with a high pass filter $3 \mathrm{kHz}$ and stimulus repetitions 1,500 and found better threshold in active compound groups (Genta-w SAMC, Genta-w DD, and Genta-w SAC) than the controls. Presumably it would be wiser if the high pass filter was chosen in the higher values but still we could demonstrate the beneficial effect of the compounds. Also it would be a more comprehensive study if we could add the investigation of the in- 
ner hair cells to experiment design. As a conclusion, SAMC, DD, and SAC are derivative of garlic seems to attenuate aminoglycoside-induced hearing loss. The effect of SAMC and DD seems to be more prominent than that of SAC.

\section{CONFLICT OF INTEREST}

No potential conflict of interest relevant to this article was reported.

\section{ACKNOWLEDGMENTS}

This study was presented at Young Otolaryngologist day in 11th Annual Middle East Update in Otolaryngology-Conference \& Exhibition-Head and Neck Surgery, April 19th-21st 2014, Dubai, UAE as an oral presentation. SAMC, DD, and SAMC were generous gift of Wakunaga Pharmaceutical Co. Ltd. (Hiroshima, Japan).

\section{REFERENCES}

1. Schacht J. Aminoglycoside ototoxicity: prevention in sight? Otolaryngol Head Neck Surg. 1998 May;118(5):674-7.

2.WuWJ, Sha SH, Schacht J. Recent advances in understanding aminoglycoside ototoxicity and its prevention. Audiol Neurootol. 2002 May-Jun;7(3):171-4.

3. Oliveira JA, Canedo DM, Rossato M, Andrade MH. Self-protection against aminoglycoside ototoxicity in guinea pigs. Otolaryngol Head Neck Surg. 2004 Sep;131(3):271-9.

4. Feldman L, Efrati S, Eviatar E, Abramsohn R, Yarovoy I, Gersch E, et al. Gentamicin-induced ototoxicity in hemodialysis patients is ameliorated by N-acetylcysteine. Kidney Int. 2007 Aug;72(3):359-63.

5. Maldonado PD, Barrera D, Medina-Campos ON, Hernandez-Pando R, Ibarra-Rubio ME, Pedraza-Chaverri J. Aged garlic extract attenuates gentamicin induced renal damage and oxidative stress in rats. Life Sci. 2003 Oct;73(20):2543-56.

6. Pedraza-Chaverri J, Maldonado PD, Medina-Campos ON, OlivaresCorichi IM, Granados-Silvestre MA, Hernandez-Pando R, et al. Garlic ameliorates gentamicin nephrotoxicity: relation to antioxidant enzymes. Free Radic Biol Med. 2000 Oct;29(7):602-11.

7.Takumida M, Popa R,Anniko M. Free radicals in the guinea pig inner ear following gentamicin exposure. ORL J Otorhinolaryngol Relat Spec. 1999 Mar-Apr;61(2):63-70.

8. Sergi B, Fetoni AR, Ferraresi A, Troiani D, Azzena GB, Paludetti G, et al. The role of antioxidants in protection from ototoxic drugs. Acta Otolaryngol Suppl. 2004 May;(552):42-5.

9. Rietz B, Isensee H, Strobach H, Makdessi S, Jacob R. Cardioprotec- tive actions of wild garlic (allium ursinum) in ischemia and reperfusion. Mol Cell Biochem. 1993 Feb;119(1-2):143-50.

10. Singh SP, Abraham SK, Kesavan PC. In vivo radioprotection with garlic extract. Mutat Res. 1995 Dec;345(3-4):147-53.

11. Ide N, Matsuura H, Itakura Y. Scavenging effect of aged garlic extract and its constituents on active oxygen species. Phytother Res. 1996 Jun;10(4):340-1.

12. Pedraza-Chaverri J, Gonzalez-Orozco AE, Maldonado PD, Barrera D, Medina-Campos ON, Hernandez-Pando R. Diallyl disulfide ameliorates gentamicin-induced oxidative stress and nephropathy in rats. Eur J Pharmacol. 2003 Jul;473(1):71-8.

13. Maldonado PD, Barrera D, Rivero I, Mata R, Medina-Campos ON, Hernandez-Pando R, et al. Antioxidant S-allylcysteine prevents gentamicin-induced oxidative stress and renal damage. Free Radic Biol Med. 2003 Aug;35(3):317-24.

14. Iqbal M, Athar M. Attenuation of iron-nitrilotriacetate (Fe-NTA)mediated renal oxidative stress, toxicity and hyperproliferative response by the prophylactic treatment of rats with garlic oil. Food Chem Toxicol. 1998 Jun;36(6):485-95.

15. Pedraza-Chaverri J, Barrera D, Maldonado PD, Chirino YI, MaciasRuvalcaba NA, Medina-Campos ON, et al. S-allylmercaptocysteine scavenges hydroxyl radical and singlet oxygen in vitro and attenuates gentamicin-induced oxidative and nitrosative stress and renal damage in vivo. BMC Clin Pharmacol. 2004 Apr;4:5.

16. Uzun L, Balbaloglu E, Akinci H. Garlic-supplemented diet attenuates gentamicin-induced ototoxicity: an experimental study. Ann Otol Rhinol Laryngol. 2012 Feb;121(2):139-43.

17. Seckiner I, Bayrak O, Can M, Mungan AG, Mungan NA. Garlic supplemented diet attenuates gentamicin nephrotoxicity in rats. Int Braz J Urol. 2014 Jul-Aug;40(4):562-7.

18. Rybak LP, Ramkumar V. Ototoxicity. Kidney Int. 2007 Oct;72(8): 931-5.

19. Amagase H. Clarifying the real bioactive constituents of garlic. J Nutr. 2006 Mar;136(3 Suppl):716S-725S.

20. Guo Z, Muller D, Pentz R, Kress G, Siegers CP. Bioavailability of sulphur-containing ingredients of garlic in the rat. Planta Med. 1990;56: 692-3.

21. Lachmann G, Lorenz D, RadeckW, Steiper M.The pharmacokinetics of the S35 labeled labeled garlic constituents alliin, allicin and vinyldithiine. Arzneimittelforschung. 1994 Jun;44(6):734-43.

22. Xiao D, Pinto JT, Soh JW, Deguchi A, Gundersen GG, Palazzo AF, et al. Induction of apoptosis by the garlic-derived compound S-allylmercaptocysteine (SAMC) is associated with microtubule depolymerization and c-Jun NH(2)-terminal kinase 1 activation. Cancer Res. 2003 Oct;63(20):6825-37.

23. Sumioka I, Matsura T, Kasuga S, Itakura Y,Yamada K. Mechanisms of protection by S-allylmercaptocysteine against acetaminophen-induced liver injury in mice. Jpn J Pharmacol. 1998 Oct;78(2):199207.

24. Sumioka I, Matsura T, Yamada K. Therapeutic effect of S-allylmercaptocysteine on acetaminophen-induced liver injury in mice. Eur J Pharmacol. 2001 Dec;433(2-3):177-85.

25. Egen-Schwind C, Eckard R, Kemper FH. Metabolism of garlic constituents in the isolated perfused rat liver. Planta Med. 1992 Aug;58 (4):301-5. 